\title{
The Role of Health Concerns in Phishing Susceptibility: Survey Design Study
}

Mohamed Abdelhamid, PhD

Department of Information Systems, College of Business, California State University Long Beach, Long Beach, CA, United States

\section{Corresponding Author:}

Mohamed Abdelhamid, PhD

Department of Information Systems

College of Business

California State University Long Beach

1250 Bellflower Boulevard

Long Beach, CA, 90840

United States

Phone: 15629852361

Email: mohamed.abdelhamid@csulb.edu

\section{Abstract}

Background: Phishing is a cybercrime in which the attackers usually impersonate a trusted source. The attackers usually send an email that contains a link that allows them to steal the receiver's personal information. In the United States, phishing is the number one cybercrime by victim count according to the Federal Bureau of Investigation's 2019 internet crime report. Several studies investigated ways to increase awareness and improve employees' resistance to phishing attacks. However, in 2019, successful phishing attacks continued to rise at a high rate

Objective: The objective of this study was to investigate the influence of personality-based antecedents on phishing susceptibility in a health care context.

Methods: Survey data were collected from participants through Amazon Mechanical Turk to test a proposed conceptual model using structural equation modeling.

Results: A total of 200 participants took part. Health concerns, disposition to trust, and risk-taking propensity yielded higher phishing susceptibility. This highlights the important of personality-based factors in phishing attacks. In addition, females had a higher phishing susceptibility than male participants

Conclusions: While previous studies used health concerns as a motivator for contexts such as sharing personal health records with providers, this study shed light on the danger of higher health concerns in enabling the number one cybercrime.

(J Med Internet Res 2020;22(5):e18394) doi: $10.2196 / 18394$

\section{KEYWORDS}

phishing; health concerns; disposition to trust; risk-taking propensity; cybercrime; security, internet; trust; risk-taking; crime victims

\section{Introduction}

\section{Background}

Phishing refers to an internet cybercrime where a normal computer or mobile phone user is targeted by a cybercriminal through email. This communication is mostly intended to lure the user to provide their sensitive data such as personal health care records, passwords, bank information, and passwords. In a phishing attack, the attacker sends an email that impersonates a legitimate organization or person. The cybercriminal uses social engineering techniques to encourage the receiver to click on a suspicious link. The link can download a malicious app or provide a form that asks the recipient to enter sensitive personal information. Phishing attacks can target individuals, employees, corporations, or governments. Attackers are motivated by many factors such as achieving financial benefits or gaining a reputation in the cybercriminal community. An attacker can sell a stolen personal health care record for thousands of dollars [1].

According to the US Federal Bureau of Investigation's annual Internet Crime Complaint Center report for 2019 [2], there were about US $\$ 3.5$ billion in financial losses alone due to cases of theft, fraud, and exploitation on the internet. The report states 
that the most prevalent type of attack was the category Phishing/Vishing/Smishing/Pharming, various terms that are used to define different means to phish. For example, fraud conducted over the phone is termed vishing. Smishing refers to attacks conducted using texting. Phishing was ranked first by the number of victims, with more than twice as many victims as the second-ranked type of attacks. Phishing attacks have been increasing significantly in the past few years [3].

A recent IBM Security report ranked the health care industry first in term of the average cost of a data breach [4]. The report stated that phishing was one of the most common methods used in carrying out an attack. In 2018, about 15 million patients' records were breached in the United States. In addition, in the first 6 months of 2019, 25 million patients were compromised with phishing being the main factor behind most breaches [5].

Phishing not only has a negative influence on individuals' monetary assets but also builds doubt every single time they are contacted via phone calls, texts, or emails. For an individual, this shatters the reliability of electronic media to carry out a variety of tasks. This poses a great danger to digitizing traditional paper-based patient records.

According to various recent reports and research, the most common features of phishing emails are that they are astonishingly true, and have a sense of urgency (pushing the recipient to take an action as soon as possible), too many links, unexpected attachments, and an anonymous or unknown sender. Researchers and experts have been developing and testing ways of raising users' awareness so that they can detect phishing attacks.

However, efforts in raising awareness are yet to prove successful. As mentioned above, the success rate of the attacks has increased globally. About 35\% of individuals do not even know what phishing is [6]. Many recent studies and current training focus on recommendations that many attackers get around. For example, Jensen et al [7] focused on training and recommendations that help individuals to avoid phishing. One of the main recommendations was to look for "https" in the address bar. Many online materials and articles list similar recommendations, including an unknown sender, generic greetings, and grammatical mistakes. However, according to a 2019 phishing trends and intelligence report, about $50 \%$ of phishing attacks use secure socket layer, making them harder to detect [8].

More importantly, attackers have constantly advanced and changed strategies. In fact, 2019 was a year of phishing evolution. Microsoft released a report in December 2019 that talked about evolving methods of phishing and explained the 3 most notable attack techniques of phishing they observed with their Microsoft Threat Protection services in 2019 [9].

Phishing is ultimately a social tactic. According the Verizon data breach investigation report [10], 43\% of cyber attacks encompass social tactics, and of those that use social tactics, $93 \%$ are phishing attacks. Therefore, constant efforts should be invested in understanding the social and personal characteristics of individuals and victims. Detection, awareness, and training strategies need to be constantly evolving. In addition, one-size-fits-all recommendations and strategies will not benefit most individuals, organizations, or industries.

\section{Literature Review}

Research related to phishing can be categorized into two main streams. The first stream of research investigates technical aspects that can automatically detect phishing attacks using various methods such as machine learning and text mining. Jain and Richariya [11] proposed that a Web browser can also be trained to screen emails.

Methods such as text clustering, text mining, topic modelling, and classification have also been used to improve systems that detect and block phishing emails. For example, Basavaraju and Prabhakar [12] used cluster analysis to detect spam emails. Jeeva and Rajsingh [13] applied association rule mining techniques to detect phishing emails that contain malicious links.

Niakanlahiji et al [14] proposed a framework of machine learning to detect phishing webpages. The framework used 15 novel features that can be used on a webpage effectively without relying on search engines or other services. Some researchers have focused on improving text mining and data extraction techniques, which are then used as scripts to extract data from emails in a semiautomatic manner and analyze them to find patterns and other data [15].

However, in 2019, about $30 \%$ of phishing emails still bypassed security detection measures [16]. Phishing volume increased by approximately $41 \%$ in 2018 , and the success rate of attacks has also increased. Although phishing detection technology is advancing, it cannot keep up with the advancement rate of phishing attacks. Therefore, many experts and researchers emphasize the importance of improving user awareness and training in regard to phishing. Most experts agree that the best way to defend against phishing attacks is to train employees and individuals to detect phishing emails in addition to security measures that detect some of the attacks automatically.

Therefore, the second stream of research has focused on the user side of the equation. User awareness of phishing emails stands to be one of the main prevention measures for phishing all around cyberspace. Hence, various awareness campaigns such as public announcements, seminars, and podcasts help to make users aware of these attacks and hence prevent phishing attacks in any form [17]. Miranda [18] suggested that phishing training programs can increase employee resistance to phishing attacks. Various studies proposed a game design framework to prevent many phishing attacks $[3,19]$. This framework enhances user avoidance behavior through motivation and hence results in preventing these phishing attacks. Other researchers looked at the characteristics of victims such as their email habits, perception of risk, and self-efficacy [20,21].

However, researchers have defined phishing as social engineering where an attacker attempts to fraudulently acquire sensitive information from a victim by impersonating a trustworthy third party. This brings in the scope for understanding normal users and their mentality gaps. Individuals are not always driven by rational thought and knowledge when making a decision or taking an action [22]. People may make 
decisions quickly and may be driven by emotions or other factors [23]. For example, Jalali et al [24] found that perceived risk is not associated with click behavior, whereas workload is positively associated with click behavior. The limited number of studies looking at the influence of individuals' characteristics and traits on phishing susceptibility in a health care context creates a gap in the research.

\section{Objective}

This study, motivated by improving phishing training and prevention measures by understanding individual characteristics, investigated the influence of personality-based factors-health concerns, disposition to trust, and risk taking-on phishing sustainability in health care-related attacks. The antecedents cover three areas of personality related to the context: concerns,

Figure 1. Conceptual model.

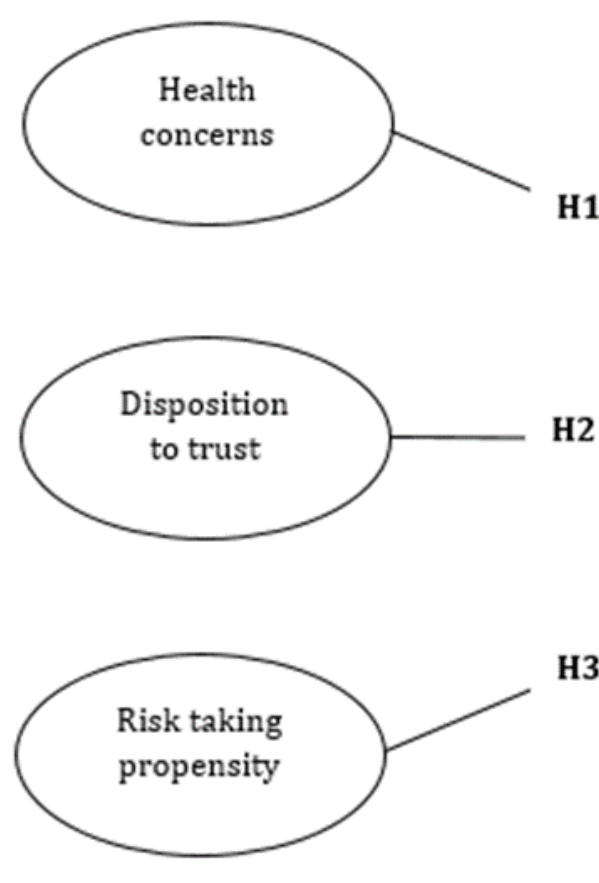

\section{Health Concerns}

Health concerns refers to the degree to which an individual is concerned about their health [25]. Health-related traits have been shown to influence individuals' choices related to behaviors affecting their health directly or indirectly [26]. People who are concerned about their health are anticipated to pursue resources as they participate in behaviors to protect their health [25]. In the context of privacy, health concerns act as a promotor to seek better health outcomes [27]. In addition, the concept of health concerns is linked with a higher likelihood to seek health information online [28]. In general, Brelsford et al [29] found that patients with a high level of health concerns are motivated to take actions related to their health. However, the construct of health concerns has not been investigated in the context of phishing.

The first study hypothesis (H1, Figure 1) was that health concerns is positively associated with phishing susceptibility.

risk, and trust. This allowed for identifying risk groups and understanding normal individuals and the mentality loopholes that an attacker uses to execute their tasks. This is one of the few studies that focused on personality-based antecedents as they relate to phishing susceptibility, specifically in the context of health care.

\section{Methods}

\section{Conceptual Model}

The following subsections argue for the relationships in the conceptual model shown in Figure 1. This is one the first studies that focused on personality-based variables and their influence on phishing susceptibility in the health care context.

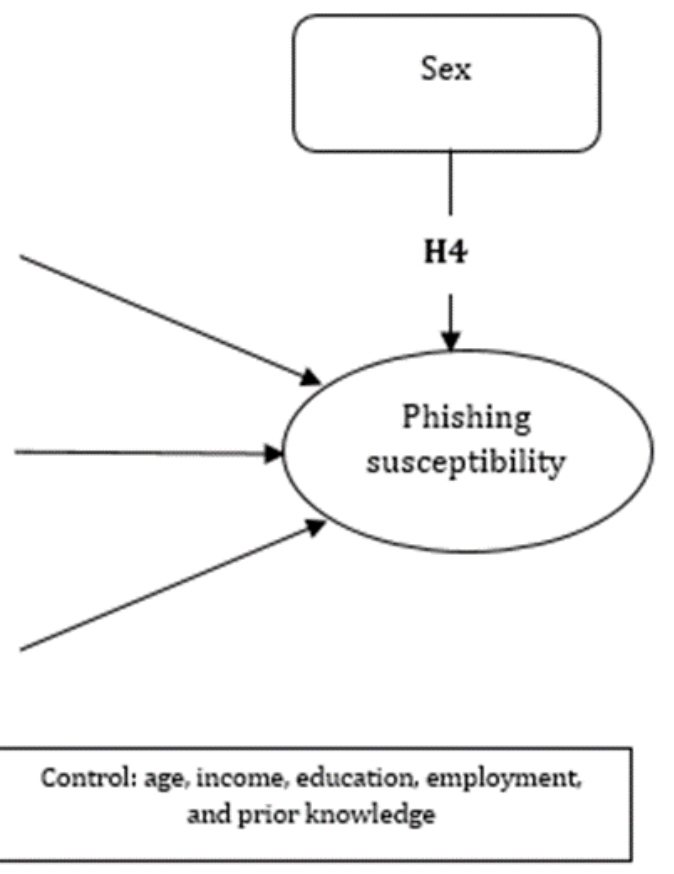

\section{Disposition to Trust}

Disposition to trust is a personality construct that refers to individuals' propensity to trust or distrust others [30]. In general, various types of trust have been extensively studied in different disciplines. The perception of trust can be linked with people or systems [31]. For example, the direct relationship between trust beliefs and use of technology has been well established [32-34]. The association between trust and information sharing has also been empirically demonstrated in previous studies [35-37]. These findings suggest that releasing personal information in exchange for e-services requires a great deal of trust. In the context of e-commerce and social media, prior literature has confirmed a positive association between trust in the system and a willingness to use the system [38,39].

The role of disposition to trust as a personality construct has been investigated in internet and website usage in general [30,40]. Bélanger and Carter [32] found that trust has an important role in promoting e-government use. Wang et al [41] investigated the effect of disposition to trust on mobile banking 
adoption. In addition, disposition to trust has been linked to a higher likelihood of deception [42].

The second study hypothesis (H2, Figure 1) was that disposition to trust is positively associated with phishing susceptibility.

\section{Risk-Taking Propensity}

A key personality aspect that influences decision making is the individual's propensity to take on risks. An individual's risk-taking personality is defined as the behavioral propensity to seek rewards despite the probability of negative consequences [43]. This construct represents an attitude that is independent of any probability of outcome, but is anchored in how individuals value those outcomes [44]. Research has shown the link between different levels of risk-taking propensities and decision making in many contexts. Though the importance of individual differences in decision making has been examined in other fields, little information security research has investigated the impact of individual traits, especially in phishing susceptibility as it relates to health care. Hansen et al [45] found that risk-taking propensity has a direct positive influence on behavioral intention in the context social media use.

The third study hypothesis (H3, Figure 1) was that risk-taking propensity is positively associated with phishing susceptibility.

\section{Role of Sex}

Differences between males and females in their online health information sharing behavior is another problem worth investigating. Male-female differences in behavior with regard to phishing has been mostly investigated in general but not specifically in health care-related phishing scenarios. For example, Sun et al [46] found that male students score better than female students in antiphishing behavior. Similarly, Verkijika [47] reported consistent findings in a general phishing context. Results are expected to be consistent in the health care phishing scenario as well.

The fourth study hypothesis (H4, Figure 1) was that males have lower phishing susceptibility than females.

\section{Data Collection}

Data were collected online by administering the survey through Amazon Mechanical Turk (Amazon.com, Inc, Seattle, WA, USA), an online survey administration platform that allows for recruiting participants. The participants were redirected to take the survey built in Qualtrics ${ }^{\mathrm{XM}}$ experience management software (Qualtrics). Participants had to be 18 years of age or older and reside in the United States. Many studies in the health care information technology context have used online data collection $[27,48]$. To measure phishing susceptibility (the dependent variable), participants were exposed to a scenario where they had to read an email and then indicate their intention to click on the email. The email was a phishing email in a health care context but participants were not told any information about its validity. The email was adopted from InfoSec [49].

\section{Data Description}

Data were cleaned and recoded using SAS version 9.4 (SAS Institute Inc). Responses that included missing data were removed from the final dataset.

\section{Variables in the Model}

All latent variables in the survey were borrowed from previous research and adapted to fit this study. Multimedia Appendix 1 shows the items and the source for variables in the model. All latent variable were measured using a Likert scale scored from 1 to 5 . Sex was recoded to a binary variable named Male where 1 referred to a male participant and 0 referred to a female participant. Control variables were age, income, education, employment, and prior knowledge of the phishing concept.

\section{Measurement Model}

After the data were cleaned using SAS version 9.4, IBM SPSS Amos version 25 (IBM Corporation) was used to assess the latent validity and reliability of the latent variables and the overall fit of the measurement model. Confirmatory factor analysis was used to evaluate the overall measurement model.

The validity of the variables was tested using the average variance extracted (AVE). Finally, multicollinearity was assessed using variable inflation factor.

\section{Results}

\section{Participant Characteristics}

After the data cleaning process, a total of 200 valid response were included in the study. Table 1 shows the descriptive statistics of the participants. For example, $43 \%$ of the participants are female and $57 \%$ are male. 
Table 1. Descriptive statistics of participants ( $\mathrm{N}=200)$.

\begin{tabular}{|c|c|}
\hline Variable, Category & Values, $\mathrm{n}(\%)$ \\
\hline \multicolumn{2}{|l|}{ Sex } \\
\hline Female & $86(43.0)$ \\
\hline Male & $114(57.0)$ \\
\hline \multicolumn{2}{|l|}{ Age (years) } \\
\hline $18-25$ & $25(12.5)$ \\
\hline $26-35$ & $113(56.5)$ \\
\hline $36-45$ & $31(15.5)$ \\
\hline $46-55$ & $19(9.5)$ \\
\hline$>55$ & $12(6.0)$ \\
\hline \multicolumn{2}{|l|}{ Education } \\
\hline High school or less & $23(11.5)$ \\
\hline Technical or community college & $26(13.0)$ \\
\hline 4-year college degree & $105(52.5)$ \\
\hline Master's degree & $42(21.0)$ \\
\hline Doctoral degree & $2(1.0)$ \\
\hline Other & $2(1.0)$ \\
\hline \multicolumn{2}{|l|}{ Income (US \$) } \\
\hline$<25,000$ & $41(20.5)$ \\
\hline 25,000 to $<50,000$ & $74(37.0)$ \\
\hline 50,000 to $<75,000$ & $58(29.0)$ \\
\hline$\geq 75,000$ & $27(13.5)$ \\
\hline
\end{tabular}

\section{Structural Equation Modeling}

Structural equation modeling (SEM) was used to test the were used to test the research hypotheses. The model explains $36.9 \%$ of the variance in phishing susceptibility. Figure 2 shows hypothesized model. Estimates derived from the SEM analysis

the SEM results for the hypothesized model.

Figure 2. Structural equation modeling (SEM) results. CFI: comparative fit index; RMSEA: root mean square error of approximation; TLI: Tucker-Lewis index.

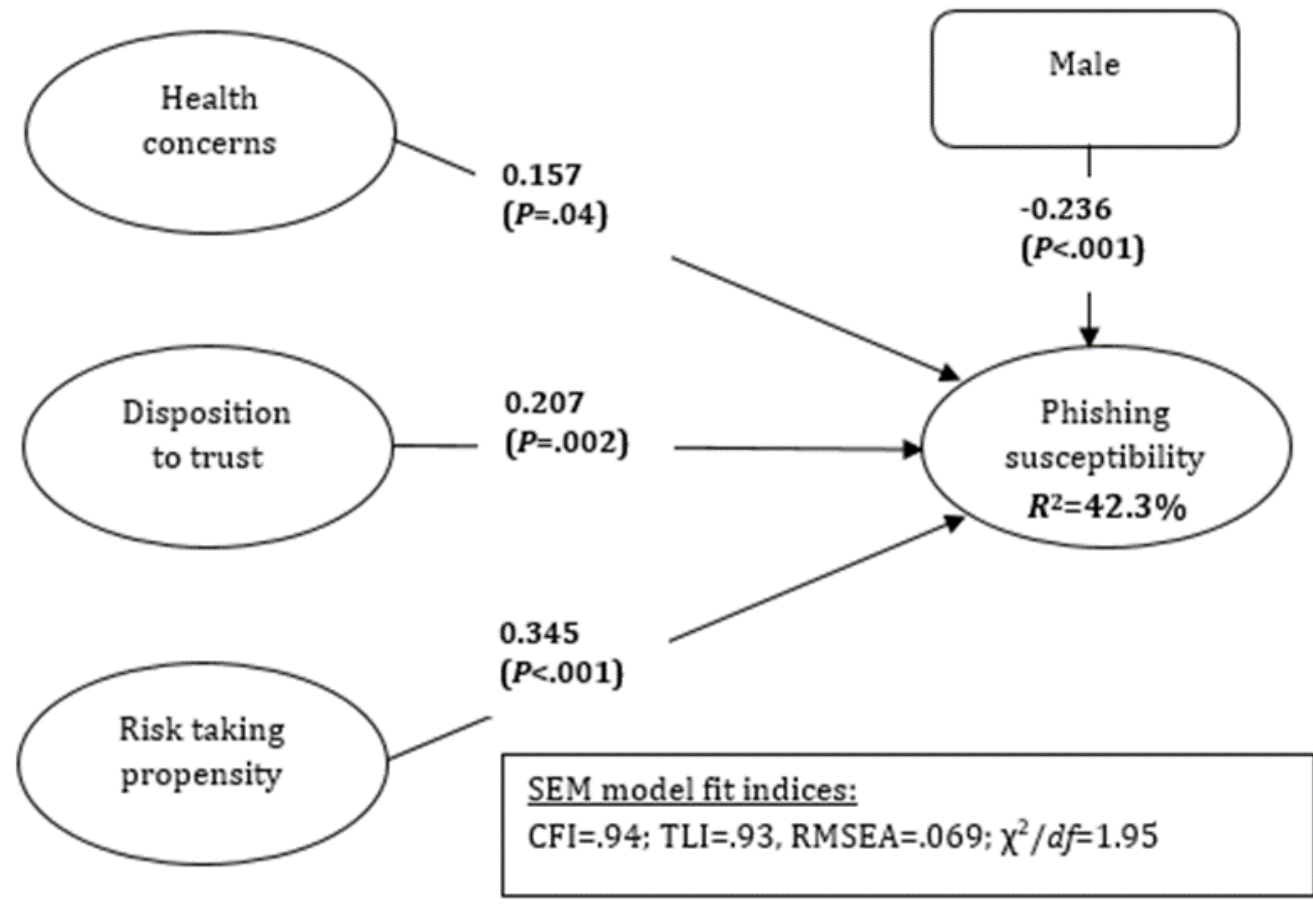


Table 2 show the results of the confirmatory factor analysis. The results show that the model fit well: root mean square error of approximation was .061, comparative fit index was .98, and Tucker-Lewis index was .97 [50]. In addition, all item loadings were high and significant, with scores ranging between .75 and .95 .

All scores exceeded .5 AVE, which meets the cutoff for establishing convergent validity [51]. In fact, the smallest AVE score was .62. All AVE scores exceeded the squared construct intercorrelation for the corresponding variable, thus establishing discriminant validity [51]. All variables were reliable, as the construct reliability scores ranged from .83 to .96 , exceeding the cutoff score of .7 for establishing reliability of the variable [52] (Table 2).

All variable inflation factor scores were well below the cutoff value of 10 [53]. Thus, there was no evidence to suggest the existence of multicollinearity. In addition, all variables were conceptually distinct.

Hypothesis 1 proposed a positive relationship between health concerns and phishing susceptibility. The SEM results supported this hypothesis. The path coefficient for the health concerns was positive and significant $(\beta=.157, P<.04)$. These results suggested that health concerns lead to higher phishing susceptibility.
Hypothesis 2 suggested that disposition to trust leads to higher phishing susceptibility. The results supported this hypothesis ( $\beta=.14, P=.04)$. The path coefficients for disposition to trust were positive and significant, which provides evidence to support hypothesis 2 . The finding proposes that individuals who have a propensity to trust others are more susceptible to phishing attacks. In addition, the magnitude of the path coefficient is higher than that of health concerns, indicating a larger influence of disposition to trust than of health concerns on phishing susceptibility.

Hypothesis 3 posited a positive relationship between risk-taking propensity and phishing susceptibility. The results show that the path coefficient was positive and significant $(\beta=.345$, $P<.001$ ), suggesting that risk-taking propensity yields a higher phishing susceptibility, thus supporting hypothesis 3 . In addition, the magnitude of the path coefficient is the highest of all antecedents.

Hypothesis 4 argued that males, compared with females, have a lower phishing susceptibility. This hypothesis was supported by the results. The path coefficient for negative and significant $(\beta=-.236, P<.001)$. This result is consistent with previous research [47]. 
Table 2. Confirmatory factor analysis results.

\begin{tabular}{|c|c|c|c|c|}
\hline Variable, item ${ }^{\mathrm{a}}$ & Loading & Construct reliability & Average variance extracted & Variable inflation factor \\
\hline Phishing susceptibility (PHS) & & .959 & .887 & $\mathbf{N} / \mathbf{A}^{\mathbf{b}}$ \\
\hline PHS_1 & .947 & & & \\
\hline PHS_2 & .947 & & & \\
\hline PHS_3 & .931 & & & \\
\hline Health concerns (HC) & & .832 & .624 & 1.27 \\
\hline HC_1 & .805 & & & \\
\hline HC_2 & .809 & & & \\
\hline HC_3 & .754 & & & \\
\hline Disposition to trust (DTR) & & .89 & .73 & 1.23 \\
\hline DTR_1 & .81 & & & \\
\hline DTR_2 & .9 & & & \\
\hline DTR_3 & .85 & & & \\
\hline Risk-taking propensity (RT) & & .904 & .76 & 1.63 \\
\hline RT_1 & .832 & & & \\
\hline RT_2 & .911 & & & \\
\hline RT_3 & .87 & & & \\
\hline \multicolumn{5}{|c|}{ Measurement model goodness-of-fit indices } \\
\hline RMSEA $^{\mathrm{c}}$ & .061 & N/A & N/A & N/A \\
\hline Comparative fit index & .98 & N/A & N/A & N/A \\
\hline Tucker-Lewis index & .97 & N/A & N/A & N/A \\
\hline$\chi^{2} / d f$ & 1.737 & N/A & N/A & N/A \\
\hline
\end{tabular}

${ }^{\mathrm{a}}$ See Multimedia Appendix 1 for item details.

${ }^{b}$ N/A: not applicable.

${ }^{\mathrm{c}}$ RMSEA: root mean square error of approximation.

\section{Discussion}

\section{Principal Findings}

This study investigated the relationship between personality-based factors and phishing susceptibility as it relates to health care. Personality-based factors have been shown to be associated with action, decision, attitude, and intention in various contexts $[27,41,45]$. However, limited research has focused on these relationship in a health care phishing context. This study focused on three main personality-based antecedents: health concerns, risk-taking propensity, and disposition to trust. This is one of the few studies that focused on these factors in the phishing context.

The study found that all three factors have in important role in leading to higher phishing susceptibility in a health care scenario. These findings suggest that personality-based factors should be taken into consideration when training individuals on phishing attacks and testing their phishing susceptibility and antiphishing behavior. The influence and effectiveness of training may differ based on personality-based traits. Thus, one-size-fits-all training, simulations, and strategy might not benefit most individuals.
While health concerns were found to be a motivator to engage patients in sharing their personal health information with providers [27], in a phishing scenario health concerns led to higher phishing susceptibility. Thus, health concerns could act as a "double-edged sword." Of all personality-based factors, the results suggested that risk-taking propensity had the highest effect on increasing phishing susceptibility. This implies that future research should study the high risk taker to find optimal methods to reduce phishing susceptibility. The study also confirmed previous findings that females are more susceptible to phishing attacks, but this research confirmed the finding in a health care context.

\section{Limitations and Future Work}

This study had several limitations, which can be addressed in future studies. The data were self-reported rather than actual behavior. Real-world behavior is very difficult to capture specifically in the health care context. In future studies, the influence of customized phishing training on individuals with different levels of personality-based factors will be tested. 


\section{Conclusions}

Phishing remains a problem that continues to increase. While companies, experts, and researchers continue to develop new methods to detect phishing attacks and improve resistance to falling a victim to phishing, attackers are advancing and improving phishing attacks at a higher and a more successful rate. Microsoft named 2019 as a year of phishing evolution as attackers made innovations in both technical and social tactics. The only way to mitigate phishing attacks is get ahead of the attackers. Training and simulation should include a balance of customized material and approaches that fit the characteristics of the receiver.

This study contributes to the phishing literature by investigating personality-based factors and reporting findings that are new and important. In addition, the study contributes to the health care information technology literature by examining health care-related phishing scenarios and factors. There is limited research in the health care context that deals with phishing susceptibility.

\section{Conflicts of Interest}

None declared.

\section{Multimedia Appendix 1}

Measurement.

[DOCX File, 164 KB-Multimedia Appendix 1]

\section{References}

1. Yao M. Your electronic medical records could be worth $\$ 1000$ to hackers. Jersey City, NJ: Forbes Media LLC; 2017 Apr 14. URL: https://www.forbes.com/sites/mariyayao/2017/04/14/your-electronic-medical-records-can-be-worth-1000-to-hackers/ \#4252d5cb50cf [accessed 2020-04-13]

2. Internet Crime Complaint Center, Federal Bureau of Investigation. 2019 Internet Crime Report.: IC3; 2019. URL: https:/ /pdf.ic3.gov/2019 IC3Report.pdf [accessed 2020-04-13]

3. Goel D, Jain AK. Mobile phishing attacks and defence mechanisms: state of art and open research challenges. Comput Secur 2018 Mar;73:519-544. [doi: 10.1016/j.cose.2017.12.006]

4. IBM Security. Cost of a Data Breach Report. Traverse City, MI: Ponemon Institute LLC; 2019. URL: https://www.ibm.com/ downloads/cas/ZBZLY7KL [accessed 2020-04-13]

5. Davis J. Health IT Security. Danvers, MA: xtelligent Healthcare Media; 2019. The 10 biggest healthcare data breaches of 2019, so far URL: https://healthitsecurity.com/news/the-10-biggest-healthcare-data-breaches-of-2019-so-far [accessed 2020-04-13]

6. Wolinsky J. The cost of phishing emails to business is staggeringly high. Passaic, NJ: ValueWalk; 2019. URL: https://www. valuewalk.com/2019/07/cost-phishing-emails-infographic/ [accessed 2020-04-13]

7. Jensen ML, Dinger M, Wright RT, Thatcher JB. Training to mitigate phishing attacks using mindfulness techniques. J Manage Inf Syst 2017 Aug 17;34(2):597-626. [doi: 10.1080/07421222.2017.1334499]

8. PhishLabs. 2019 phishing trends and intelligence report: the growing social engineering threat. 2019. URL: https://info. phishlabs.com/hubfs/2019\%20PTI\%20Report/2019\%20Phishing\%20Trends\%20and\%20Intelligence\%20Report.pdf [accessed 2020-04-13]

9. Office 365 Threat Research Team. The quiet evolution of phishing.: Microsoft; 2019 Dec 11. URL: https://www. microsoft.com/security/blog/2019/12/11/the-quiet-evolution-of-phishing/ [accessed 2020-01-09]

10. 2019 data breach investigations report. Basking Ridge, NJ: Verizon; 2019. URL: https://enterprise.verizon.com/resources/ reports/2019-data-breach-investigations-report.pdf [accessed 2020-04-16]

11. Jain A, Richariya V. Implementing a web browser with phishing detection techniques. World Comput Sci Inf Technol J 2011;1(7):289-291 [FREE Full text]

12. Basavaraju M, Prabhakar DR. A novel method of spam mail detection using text based clustering approach. Int J Comput Appl 2010 Aug 10;5(4):15-25. [doi: 10.5120/906-1283]

13. Jeeva SC, Rajsingh EB. Intelligent phishing url detection using association rule mining. Hum Centric Comput Inf Sci 2016 Jul 10;6(1):10. [doi: 10.1186/s13673-016-0064-3]

14. Niakanlahiji A, Chu BT, Al-Shaer E. PhishMon: a machine learning framework for detecting phishing webpages. 2018 Presented at: IEEE International Conference on Intelligence and Security Informatics (ISI); Nov 8-10, 2018; Miami, FL, USA. [doi: 10.1109/isi.2018.8587410]

15. Zareapoor M, Seeja K. Text mining for phishing e-mail detection. In: Jain LC, Patnaik S, Ichalkaranje N, editors. Intelligent Computing, Communication and Devices. Cham, Switzerland: Springer Nature Switzerland; 2015:65-71.

16. Crane C. 20 phishing statistics to keep you from getting hooked in 2019. St Petersburg, FL: The SSL Store; 2019 Jul 24. URL: https://www.thesslstore.com/blog/20-phishing-statistics-to-keep-you-from-getting-hooked-in-2019/ [accessed 2020-04-13] 
17. Jansson K, von Solms R. Phishing for phishing awareness. Behav Inf Technol 2013 Jun;32(6):584-593. [doi: 10.1080/0144929x.2011.632650]

18. Miranda MJ. Enhancing cybersecurity awareness training: a comprehensive phishing exercise approach. Int Manage Rev 2018;14(2):5-10 [FREE Full text]

19. Chandrasekaran M, Narayanan K, Upadhyaya S. Phishing email detection based on structural properties. 2006 Presented at: 9th Annual NYS Cyber Security Conference; Jun 14-15, 2006; Albany, NY, USA.

20. Vishwanath A, Harrison B, Ng YJ. Suspicion, cognition, and automaticity model of phishing susceptibility. Commun Res 2016 Feb 10;45(8):1146-1166. [doi: 10.1177/0093650215627483]

21. Arachchilage NAG, Love S, Beznosov K. Phishing threat avoidance behaviour: an empirical investigation. Comput Hum Behav 2016 Jul;60:185-197. [doi: 10.1016/j.chb.2016.02.065]

22. Soman D. The Last Mile: Creating Social and Economic Value From Behavioral Insights. Toronto, ON: University of Toronto Press; 2015.

23. Thaler R, Sunstein C. Nudge. London, UK: Penguin; 2009.

24. Jalali MS, Bruckes M, Westmattelmann D, Schewe G. Why employees (still) click on phishing links: investigation in hospitals. J Med Internet Res 2020 Jan 23;22(1):e16775 [FREE Full text] [doi: 10.2196/16775] [Medline: 32012071]

25. Jayanti RK, Burns AC. The antecedents of preventive health care behavior: an empirical study. J Acad Mark Sci 1998 Jan 01;26(1):6-15. [doi: 10.1177/0092070398261002]

26. Moorman C, Matulich E. A model of consumers' preventive health behaviors: the role of health motivation and health ability. J Consum Res 1993 Sep;20(2):208. [doi: 10.1086/209344]

27. Abdelhamid M. Greater patient health information control to improve the sustainability of health information exchanges. J Biomed Inform 2018 Jul;83:150-158 [FREE Full text] [doi: 10.1016/j.jbi.2018.06.002] [Medline: 29894811]

28. White RW, Horvitz E. From health search to healthcare: explorations of intention and utilization via query logs and user surveys. J Am Med Inform Assoc 2014;21(1):49-55 [FREE Full text] [doi: 10.1136/amiajnl-2012-001473] [Medline: 23666794]

29. Brelsford KM, Spratt SE, Beskow LM. Research use of electronic health records: patients' perspectives on contact by researchers. J Am Med Inform Assoc 2018 Sep 01;25(9):1122-1129 [FREE Full text] [doi: 10.1093/jamia/ocy087] [Medline: 29986107]

30. Wu G, Hu X, Wu Y. Effects of perceived interactivity, perceived web assurance and disposition to trust on initial online trust. J Comput Mediat Commun 2010 Oct 1;16(1):1-26. [doi: 10.1111/j.1083-6101.2010.01528.x]

31. Gefen D, Karahanna E, Straub DW. Trust and TAM in online shopping: an integrated model. MIS Q 2003;27(1):51. [doi: 10.2307/30036519]

32. Bélanger F, Carter L. Trust and risk in e-government adoption. J Strategic Inf Syst 2008 Jun;17(2):165-176. [doi: 10.1016/j.jsis.2007.12.002]

33. Posey C, Roberts TL, Lowry PB, Bennett RJ, Courtney JF. Insiders' protection of organizational information assets: development of a systematics-based taxonomy and theory of diversity for protection-motivated behaviors. MIS Q 2013 Apr 4;37(4):1189-1210. [doi: 10.25300/misq/2013/37.4.09]

34. Hung SY, Chang CM, Yu TJ. Determinants of user acceptance of the e-government services: the case of online tax filing and payment system. Gov Inf Q 2006 Jan;23(1):97-122. [doi: 10.1016/j.giq.2005.11.005]

35. Mou J, Shin D, Cohen JF. Trust and risk in consumer acceptance of e-services. Electron Commerce Res $2015 \mathrm{Nov}$ 5;17(2):255-288. [doi: 10.1007/s10660-015-9205-4]

36. Anderson CL, Agarwal R. The digitization of healthcare: boundary risks, emotion, and consumer willingness to disclose personal health information. Inf Syst Res 2011 Sep;22(3):469-490. [doi: 10.1287/isre.1100.0335]

37. Abdelhamid M, Gaia J, Sanders GL. Putting the focus back on the patient: how privacy concerns affect personal health information sharing intentions. J Med Internet Res 2017 Sep 13;19(9):e169 [FREE Full text] [doi: 10.2196/jmir.6877] [Medline: 28903895]

38. Pavlou PA, Fygenson M. Understanding and predicting electronic commerce adoption: an extension of the theory of planned behavior. MIS Q 2006;30(1):115. [doi: 10.2307/25148720]

39. Lin WY, Zhang X, Song H, Omori K. Health information seeking in the Web 2.0 age: trust in social media, uncertainty reduction, and self-disclosure. Comput Hum Behav 2016 Mar;56:289-294. [doi: 10.1016/j.chb.2015.11.055]

40. McKnight DH, Choudhury V, Kacmar C. Developing and validating trust measures for e-commerce: an integrative typology. Inf Syst Res 2002 Sep;13(3):334-359. [doi: 10.1287/isre.13.3.334.81]

41. Wang SW, Ngamsiriudom W, Hsieh C. Trust disposition, trust antecedents, trust, and behavioral intention. Serv Ind J 2015 Jun;35(10):555-572. [doi: 10.1080/02642069.2015.1047827]

42. Wright RT, Marett K. The influence of experiential and dispositional factors in phishing: an empirical investigation of the deceived. J Manage Inf Syst 2014 Dec 08;27(1):273-303. [doi: 10.2753/mis0742-1222270111]

43. Gorka SM, Liu H, Klein D, Daughters SB, Shankman SA. Is risk-taking propensity a familial vulnerability factor for alcohol use? An examination in two independent samples. J Psychiatr Res 2015 Sep;68:54-60 [FREE Full text] [doi:

10.1016/j.jpsychires.2015.05.019] [Medline: 26228401] 
44. Simonsohn U. Direct risk aversion: evidence from risky prospects valued below their worst outcome. Psychol Sci 2009 Jun;20(6):686-692. [doi: 10.1111/j.1467-9280.2009.02349.x] [Medline: 19422629]

45. Hansen JM, Saridakis G, Benson V. Risk, trust, and the interaction of perceived ease of use and behavioral control in predicting consumers' use of social media for transactions. Comput Hum Behav 2018 Mar;80:197-206. [doi: 10.1016/j.chb.2017.11.010]

46. Sun JC, Yu S, Lin SS, Tseng S. The mediating effect of anti-phishing self-efficacy between college students' internet self-efficacy and anti-phishing behavior and gender difference. Comput Hum Behav 2016 Jun;59:249-257. [doi: 10.1016/j.chb.2016.02.004]

47. Verkijika SF. "If you know what to do, will you take action to avoid mobile phishing attacks": self-efficacy, anticipated regret, and gender. Comput Hum Behav 2019 Dec;101:286-296. [doi: 10.1016/j.chb.2019.07.034]

48. Angst CM, Agarwal R. Adoption of electronic health records in the presence of privacy concerns: the elaboration likelihood model and individual persuasion. MIS Q 2009;33(2):339. [doi: 10.2307/20650295]

49. Dalasta D. Healthcare phishing attacks. Madison, WI: InfoSec; 2020. URL: https://resources.infosecinstitute.com/category/ enterprise/phishing/the-phishing-landscape/phishing-attacks-by-demographic/phishing-in-healthcare/\#gref [accessed 2020-04-13]

50. Hu L, Bentler PM. Cutoff criteria for fit indexes in covariance structure analysis: conventional criteria versus new alternatives. Struct Equation Modeling 1999 Jan;6(1):1-55. [doi: 10.1080/10705519909540118]

51. Fornell C, Larcker DF. Structural equation models with unobservable variables and measurement error: algebra and statistics. J Mark Res 2018 Nov 28;18(3):382-388. [doi: 10.1177/002224378101800313]

52. Joreskog K, Sorbom D. LISREL 8 User's Reference Guide. Mooresville, IN: Scientific Software; 1989.

53. Tabachnick B, Fidell L. Using Multivariate Statistics. Boston, MA: Pearson Higher Education; 2013.

\section{Abbreviations \\ AVE: average variance extracted \\ SEM: structural equation modeling}

Edited by G Eysenbach; submitted 24.02.20; peer-reviewed by S Guness; comments to author 16.03.20; revised version received 29.03.20; accepted 30.03.20; published 04.05.20

\section{Please cite as:}

Abdelhamid $M$

The Role of Health Concerns in Phishing Susceptibility: Survey Design Study

J Med Internet Res 2020;22(5):e18394

URL: https://www.jmir.org/2020/5/e18394

doi: $\underline{10.2196 / 18394}$

PMID: 32364511

CMohamed Abdelhamid. Originally published in the Journal of Medical Internet Research (http://www.jmir.org), 04.05.2020. This is an open-access article distributed under the terms of the Creative Commons Attribution License (https://creativecommons.org/licenses/by/4.0/), which permits unrestricted use, distribution, and reproduction in any medium, provided the original work, first published in the Journal of Medical Internet Research, is properly cited. The complete bibliographic information, a link to the original publication on http://www.jmir.org/, as well as this copyright and license information must be included. 